\title{
Effect of negative permittivity and permeability on the transmission of electromagnetic waves through a structure containing left-handed material
}

\author{
Muin F. Ubeid ${ }^{1 *}$, Mohammed M. Shabat ${ }^{1}$, Mohammed O. Sid-Ahmed ${ }^{2}$ \\ ${ }^{1}$ Department of Physics, Faculty of Science, Islamic University of Gaza, Gaza, Palestine; \\ *Corresponding Author: mubeid@mail.iugaza.edu \\ ${ }^{2}$ Department of Physics, Faculty of Science, Sudan University of Science and Technology, Khartoum, The Republic of The Sudan
}

Received 5 March 2011; revised 23 March 2011; accepted 27 March 2011.

\begin{abstract}
We investigate the characteristics of electromagnetic wave reflection and transmission by multilayered structures consisting of a pair of left-handed material (LHM) and dielectric slabs inserted between two semi-infinite dielectric media. The theoretical aspect is based on Maxwell's equations and matching the boundary conditions for the electric and magnetic fields of the incident waves at each layer interface. We calculate the reflected and transmitted powers of the multilayered structure taking into account the widths of the slabs and the frequency dependence of permittivity and permeability of the LHM. The obtained results satisfy the law of conservation of energy. We show that if the semi-infinite dielectric media have the same refractive index and the slabs have the same width, then the reflected (and transmitted) powers can be minimized (and maximized) and the powers-frequency curves show no ripple. On the other hand if the semi-infinite dielectric media have different values of refractive indices and the slabs have different widths, then under certain conditions the situation of minimum and maximum values of the mentioned powers will be reversed.
\end{abstract}

Keywords: Electromagnetic Waves; Left-Handed Materials; Frequency; Reflected and Transmitted Powers

\section{INTRODUCTION}

Metamaterials (sometimes termed left-handed materials (LHMs)) are materials whose permittivity $\varepsilon$ and permeability $\mu$ are both negative and consequently have negative index of refraction. These materials are artificial and theoretically discussed first by Veselago [1] over 40 years ago. The first realization of such materials, consisting of split-ring resenators (SRRs) and continuous wires, was first introduced by Pendry [2,3]. Regular materials are materials whose $\varepsilon$ and $\mu$ are both positive and termed right handed materials (RHMs). R. A. Shelby et al. [4] have studied negative refraction in LHMs. I. V. Shadrivov [5] has investigated nonlinear guided waves in LHMs. N. Garcia et al. [6] have shown that LHMs don't make a perfect lens. Kong [7] has provided a general formulation for the electromagnetic wave interaction with stratified metamaterial structures. M. M. Shabat et al [8] have discussed Nonlinear TE surface waves in a left-handed material and magnetic super lattice waveguide structure. I. Kourakis et al. [9] have investigated a nonlinear propagation of electromagnetic waves in negative-refraction index LHM. H. Cory et al. [10] and C. Sabah et al. [11] have estimated high reflection coatings of multilayered structure. Oraizi et al. [12] have obtained a zero reflection from multilayered metamaterial structures.

In this paper we consider a structure consisting of LHM and dielectric slabs inserted between two semiinfinite dielectric media. A plane polarized wave is obliquely incident on it. We use Maxwell's equations and match the boundary conditions for the electric and magnetic fields of the incident waves at each layer interface. Then we solve the obtained equations for the unknown parameters to calculate the reflection and transmission coefficients. We take into account the frequency dependence of permittivity and permeability of the LHM (in contradict with $[10,11]$ ), widths of the slabs, refractive indices of the media and angle of incidence of the incident waves. Maximum and minimum transmitted (minimum and maximum reflected) powers of the considered structure are proposed. The numerical results are 
in agreement with the law of conservation of energy given by $[10,13]$. It is found that the numerical results of Figure 3 (the case $a \neq b$ ) is similar to Figure 4(b) obtained by [14], this is another evidence for validity of the performed computations.

The paper is organized as follows: our theory is formulated in section 2. Numerical results and applications are described in section 3. Our conclusions are presented in section 4 .

\section{THEORY}

Consider four regions each with permittivity $\varepsilon_{i}$ and permeability $\mu_{i}$, where $i$ represents the region order. Region 1 is a vacuum $\left(\varepsilon_{1}=\varepsilon_{o}, \mu_{1}=\mu_{o}\right)$, Region 2 is a regular dielectric $\left(\varepsilon_{2}, \mu_{2}\right)$, Region 3 is a metamaterial $\left(\left(\varepsilon_{3}(\omega)\right), \mu_{3}(\omega)\right)$, Region 4 is a regular dielectric $\left(\varepsilon_{4}, \mu_{4}\right)$. A polarized plane wave in Region 1 incident on the plane $\mathrm{z}=0$ at some angle $\theta$ relative to the normal to the boundary (see Figure 1).

The electric field of the incident wave in Region 1 can be written as [7-10]:

$$
\boldsymbol{E}_{1}=\left(A \mathrm{e}^{i k_{1 z} z}+B \mathrm{e}^{-i k_{1 z} z}\right) \mathrm{e}^{i\left(k_{1 x} x-\omega t\right)} \hat{y}
$$

To find the corresponding magnetic field $\boldsymbol{H}_{1}$, we start with Maxwell's equation [15]:

$$
\nabla \times \boldsymbol{E}_{1}=-\frac{\partial \boldsymbol{B}_{1}}{\partial t}
$$

substituting $\boldsymbol{B}_{1}=\mu_{1} \boldsymbol{H}_{1}$ and solving for $\boldsymbol{H}_{1}$ yield:

$$
\begin{aligned}
\boldsymbol{H}_{1}= & \frac{1}{\mu_{1} \omega}\left[\left(A k_{1 x} \mathrm{e}^{i k_{1 z} z}+B k_{1 x} \mathrm{e}^{-i k_{1 z} z}\right) \hat{z}\right. \\
& \left.+\left(-A k_{1 z} \mathrm{e}^{i k_{1 z} z}+B k_{1 z} \mathrm{e}^{-i k_{1 z} z}\right) \hat{x}\right] \mathrm{e}^{i\left(k_{1 x} x-\omega t\right)}
\end{aligned}
$$

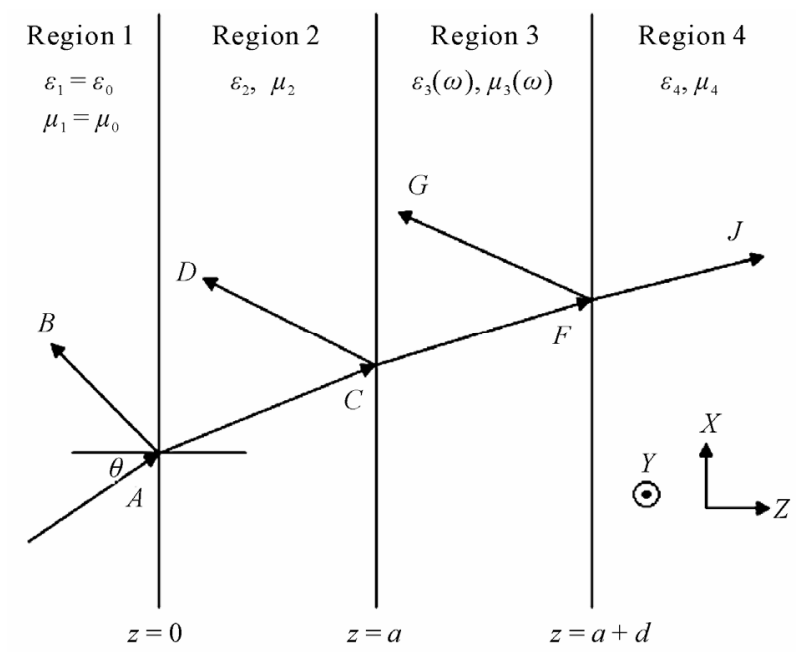

Figure 1. Wave propagation through a structure consisting of a pair of dielectric and metamaterial embedded between two dielectric semi-infinite media.
The electric and magnetic fields in Regions 2, 3 and 4 can be written in the same manner as follows:

$$
\begin{gathered}
\boldsymbol{E}_{2}=\left(C e^{i k_{2 z} z}+D e^{-i k_{2 z} z}\right) \mathrm{e}^{i\left(k_{2 x} x-\omega t\right)} \hat{y} \\
\boldsymbol{H}_{2}=\frac{1}{\mu_{2} \omega}\left[\left(C k_{2 x} \mathrm{e}^{i k_{2 z} z}+D k_{2 x} \mathrm{e}^{-i k_{2 z} z}\right) \hat{z}\right. \\
\left.+\left(-C k_{2 z} \mathrm{e}^{i k_{2 z} z}+D k_{2 z} \mathrm{e}^{-i k_{2 z} z}\right) \hat{x}\right] \mathrm{e}^{i\left(k_{2 x} x-\omega t\right)} \\
\boldsymbol{E}_{3}=\left(F \mathrm{e}^{i k_{3 z} z}+G \mathrm{e}^{-i k_{3 z} z}\right) \mathrm{e}^{i\left(k_{3 x} x-\omega t\right)} \hat{y} \\
\boldsymbol{H}_{3}=\frac{1}{\mu_{3} \omega}\left[\left(F k_{3 x} \mathrm{e}^{i k_{3 z} z}+G k_{3 x} \mathrm{e}^{-i k_{3 z} z}\right) \hat{z}\right. \\
\left.+\left(-F k_{3 z} \mathrm{e}^{i k_{3 z} z}+G k_{3 z} \mathrm{e}^{-i k_{3 z} z}\right) \hat{x}\right] \mathrm{e}^{i\left(k_{3 x} x-\omega t\right)} \\
\boldsymbol{E}_{4}=J \mathrm{e}^{i k_{4 z} z} \mathrm{e}^{i\left(k_{4 x} x-\omega t\right)} \hat{y} \\
\boldsymbol{H}_{4}=\frac{1}{\mu_{4} \omega}\left(J k_{4 x} \mathrm{e}^{i k_{4 z} z} \hat{z}-J k_{4 z} \mathrm{e}^{i k_{4 z} z} \hat{x}\right) \mathrm{e}^{i\left(k_{4 x} x-\omega t\right)}
\end{gathered}
$$

where $k_{i}=n_{i} \omega / c$ is the wave vector inside the material and $n_{i}=\sqrt{\frac{\varepsilon_{i} \mu_{i}}{\varepsilon_{o} \mu_{o}}}$ is the refractive index of it.

Matching the boundary conditions for $\boldsymbol{E}$ and $\boldsymbol{H}$ fields at each layer interface, that is at $z=0, \boldsymbol{E}_{1}=\boldsymbol{E}_{2}$ and $\boldsymbol{H}_{1}=\boldsymbol{H}_{2}$, at $z=a \boldsymbol{E}_{2}=\boldsymbol{E}_{3}$ and $\boldsymbol{H}_{2}=\boldsymbol{H}_{3}$, and at $z$ $=a+d, \boldsymbol{E}_{3}=\boldsymbol{E}_{4}$ and $\boldsymbol{H}_{3}=\boldsymbol{H}_{4}$. This yields the following equations $[10,12-15]$ :

$$
\begin{gathered}
A+B=C+D \\
\frac{k_{1 z}}{\mu_{1}}(A-B)=\frac{k_{2 z}}{\mu_{2}}(C-D) \\
C \mathrm{e}^{i k_{2 z} a}+D \mathrm{e}^{-i k_{2 z} a}=F \mathrm{e}^{i k_{3 z} a}+G \mathrm{e}^{-i k_{3 z} a} \\
\frac{k_{2 z}}{\mu_{2}}\left(C \mathrm{e}^{i k_{2 z} a}-D \mathrm{e}^{-i k_{2 z} a}\right)=\frac{k_{3 z}}{\mu_{3}}\left(F \mathrm{e}^{i k_{3 z} a}-G \mathrm{e}^{-i k_{3 z} a}\right) \\
F \mathrm{e}^{i k_{3 z}(a+d)}+G \mathrm{e}^{-i k_{3 z}(a+d)}=J \mathrm{e}^{i k_{4 z}(a+d)} \\
\frac{k_{3 z}}{\mu_{3}}\left(F \mathrm{e}^{i k_{3 z}(a+d)}-G \mathrm{e}^{-i k_{3 z}(a+d)}\right)=\frac{k_{4 z}}{\mu_{4}} J \mathrm{e}^{i k_{4 z}(a+d)}
\end{gathered}
$$

Letting $A=1$ and solving these six equations for the unknown parameters enable us to calculate the reflection and transmission coefficients $\mathrm{B}$ and $\mathrm{J}$ respectively $[10,15]$. The reflected power $\mathrm{R}$ equal to the reflection coefficient $\mathrm{B}$ times its complex conjugate and the transmitted power $\mathrm{T}$ equal to the transmission coefficient $\mathrm{J}$ times its complex conjugate $[10,15]$, leading to;

$$
\begin{gathered}
R=B B^{*} \\
T=J J^{*}
\end{gathered}
$$

The law of conservation of energy is given by [10, 13]:

$$
R+\left(k_{4 z} / k_{1 z}\right) T=1
$$


where:

$$
k_{i z}=\frac{\omega}{c} \sqrt{n_{i}^{2}-n_{1}^{2} \sin ^{2} \theta}
$$

\section{NUMERICAL RESULTS AND APPLICATIONS}

For the metamaterial in region 3 we will employ a non-dispersive metamaterial with $\varepsilon$ and $\mu$ given by [2,3,12,15-17]:

$$
\begin{gathered}
\varepsilon_{3}(\omega)=1-\frac{F_{e} \omega_{e p}^{2}}{\omega^{2}-\omega_{e o}^{2}-i \gamma_{e} \omega} \\
\mu_{3}(\omega)=1-\frac{F_{m} \omega_{m p}^{2}}{\omega^{2}-\omega_{m o}^{2}-i \gamma_{m} \omega}
\end{gathered}
$$

where $\omega_{e p}$ and $\omega_{m p}$ are the electric and magnetic plasma frequencies, $\omega_{e o}$ and $\omega_{m o}$ are the electric and magnetic resonance frequencies. $F_{e}$ and $F_{m}$ are the scaling filling parameters.

We have used the following parameters in [15]: $\omega_{m p}=2 \pi 10.95 \mathrm{GHz}, \omega_{m o}=2 \pi 10.1 \mathrm{GHz}, F_{m}=0.26$, $\omega_{e p}=2 \pi 13.3 \mathrm{GHz}, \omega_{e o}=2 \pi 10.3 \mathrm{GHz}, F_{e}=0.37$, with no loss case i.e. $\gamma_{e}=\gamma_{m}=0$. In this case, the frequency range in which $\varepsilon_{3}(\omega)$ and $\mu_{3}(\omega)$ are negative extends from 10.3 up to $11.4 \mathrm{GHz}$. The obtained values of $\varepsilon_{3}(\omega), \mu_{3}(\omega)$ in addition to $k_{i z}$ are used in (10-15). These equations are solved for the parameters B and $\mathrm{J}$. Then the reflected and transmitted powers $\mathrm{R}$ and $\mathrm{T}$ can be calculated. The transmitted power is plotted as a function of $\omega$ under different conditions as follows:

The dependence of $\mu_{3}$ on $\omega$ for the metamaterial in Region 3 is taken into account [2-5,15-17], it is considered by $[10,11]$ to be $\mu_{3}=-\mu_{o}$. The difference between the two cases can be noticed from Figure 2.

The thickness of the slabs is taken to be the same $a=$ $d, n_{2}=\left|n_{3}\right|, n_{1}=n_{4}, \theta$ is kept constant. In this case the structure's reflected and transmitted powers variation with frequency are smooth and show no ripples as shown in Figure 3. To check the validity of computations for the case $a \neq b$ as an example, let $\omega=2 \pi 11 \mathrm{GHz}$, then: $\mu_{3}$ $=-0.641635071, \varepsilon_{3}=-3.3896244113, k_{4 z} / k_{1 z}=1, B=$ $-0.5709597786+2.95809086 \mathrm{i}, J=-0.7435211938+$ $0.1845247865 \mathrm{i}, R=0.4131268376, T=0.5868731625$. In this case the left hand side of (18) is equal to 0.999 99999 which verifies the law of conservation of energy. It can be realized from Figure 3 that this law is satisfied by the performed computations. The same procedure can be applied to other computations in this paper.

Low reflected and high transmitted powers can be achieved if $n_{1}=n_{4}, n_{2}=\left|n_{3}\right|$ and $a=d$ [10]. In this case $R=0$ (minimum) and $T=1$ (maximum) for any frequency and for any angle of incidence (Figure 4). If

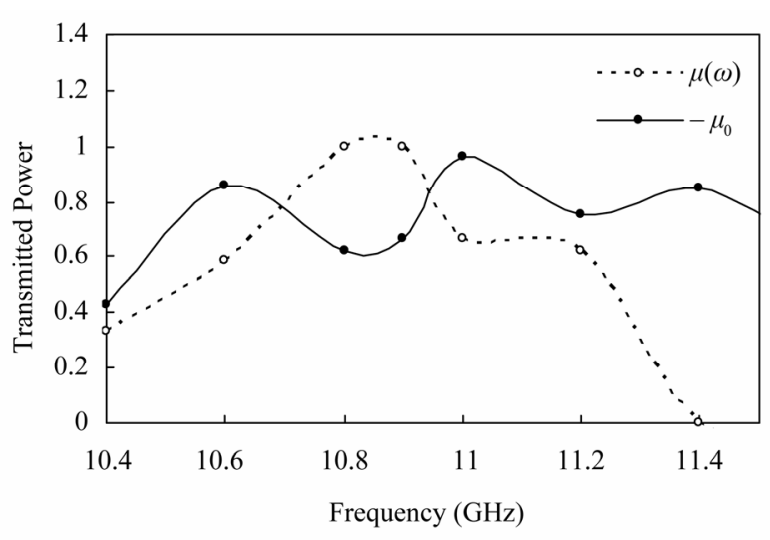

Figure 2. Transmitted power variation with frequency. Two cases are taken into account: $\mu_{3}$ is a function of $\omega, \mu_{3}$ is a con$\operatorname{stant}\left(-\mu_{0}\right)$.

$n_{1} \neq n_{4} \quad\left(\right.$ and $a=d, \quad n_{2}=\left|n_{3}\right|$ ), then both $R$ and $T$ depend on the values of the refractive indices of the initial and final media $n_{1}$ and $n_{4}$, and on the angle of incidence $\theta$ see Figures 5 and 6.

High reflected and Low transmitted powers: in order to maximize $\mathrm{R}$ and minimize $\mathrm{T}$, one has to choose a pair of adjacent dielectric and metamaterial slabs with highly contrasted refractive indices $\left(n_{1}<n_{2}, n_{2}>n_{3}, n_{3}<n_{4}\right)$ and $n_{2} a=n_{3} d=c \pi / \omega_{o} \quad[10]$, where $\omega_{o}$ is the central frequency $\left(\omega_{o}=10.9 \mathrm{GHz}\right)$. In this case by a judicious combination of metamaterial and dielectric slabs, a high-reflected and low-transmitted powers are achieved, for which the dependence of $\mathrm{R}$ and $\mathrm{T}$ on frequency and on the angle of incidence is consequently diminished (Figure 7). Note that the maximum value of $\mathrm{T}$ is 0.09 at $0^{\circ}$. This value is smaller by a factor of 11 than that obtained in Figure 4. Figure 7 is different from that obtained by $[10,11]$ in the fact that, they had used a LHM with properties invariant with frequency. In our paper, the properties of the LHM in Region 3 depends on frequency (this can be realized from Eqs.20 and 21).

\section{CONCLUSIONS}

The propagation of electromagnetic waves through multilayered structures consisting of a pair of LHM and dielectric slabs inserted between semi-infinite dielectric media has been studied. The followed method has been based on Maxwell's equations and matching the boundary conditions for the electric and magnetic fields at each interface layer. The frequency dependence of $\varepsilon$ and $\mu$ of the LHM has been taken into account. The reflected and transmitted powers have been calculated numerically. The dependence of them on various parameters has been studied. Low and high transmitted powers have been achieved for any frequency and for any angle of incidence. The law of conservation of energy 


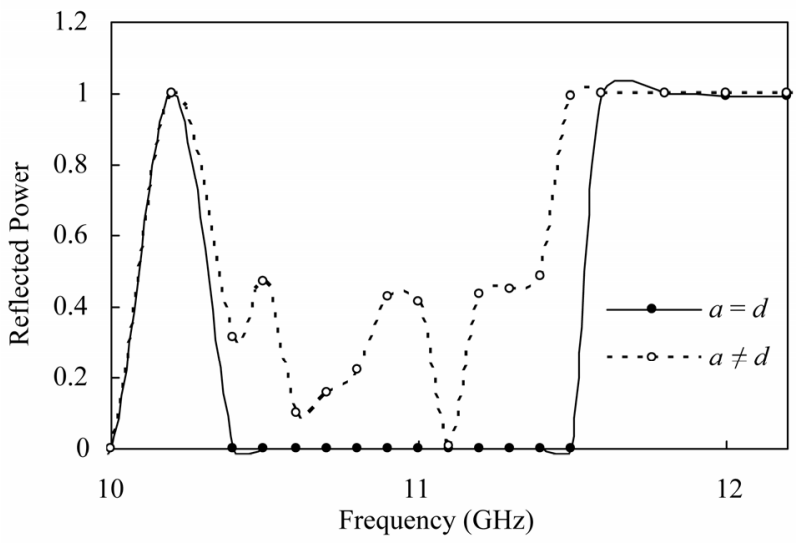

(a)

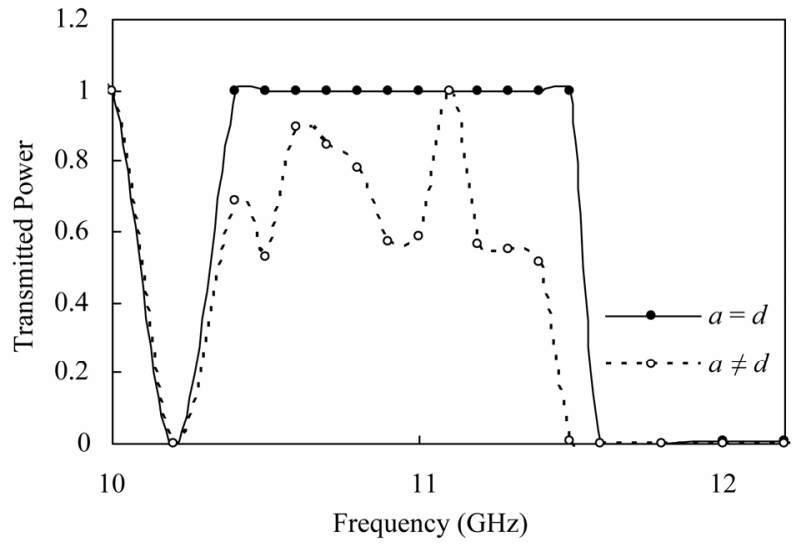

(b)

Figure 3. The reflected and Transmitted powers variation with frequency when $n_{1}=n_{4}, n_{2}=\left|n_{3}\right|$ and $\theta$ is kept constant for two cases with respect to the widths a and d of the slabs: $a=d, a \neq d$.

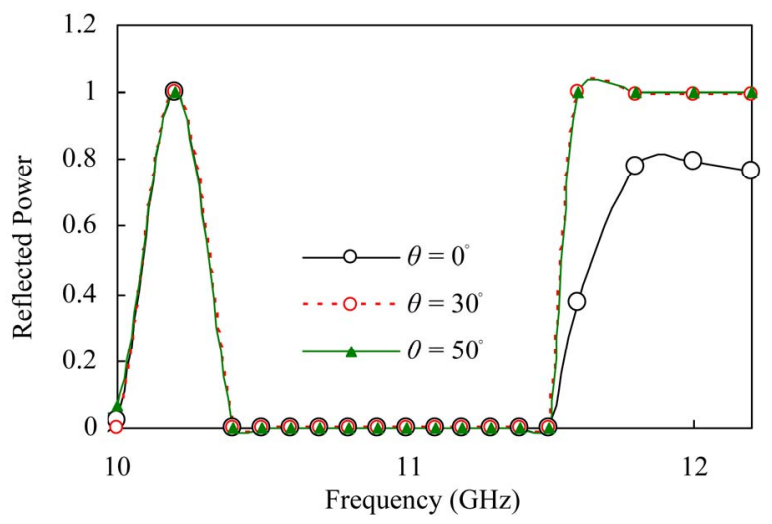

(a)

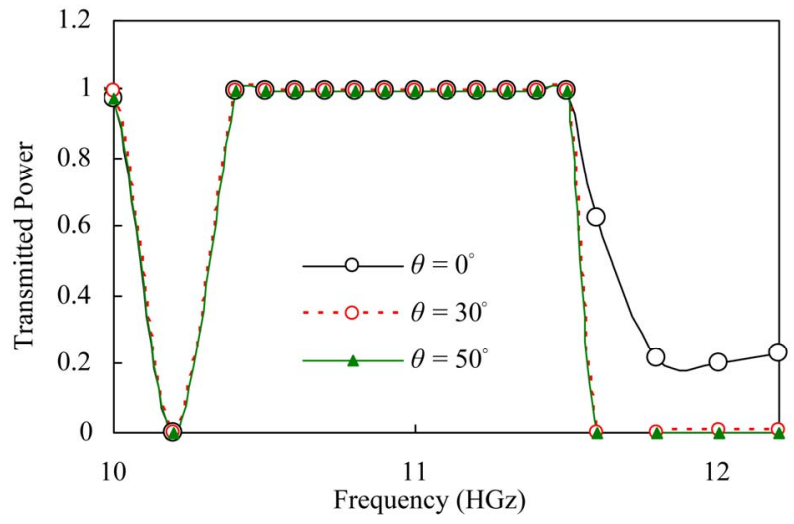

(b)

Figure 4. The reflected and transmitted powers as a function of frequency when $n_{2}=\left|n_{3}\right|, n_{1}=n_{4}$ and $a=d$ for various angle of incidence: $\theta=0^{\circ}, \theta=30^{\circ}, \theta=50^{\circ}$.

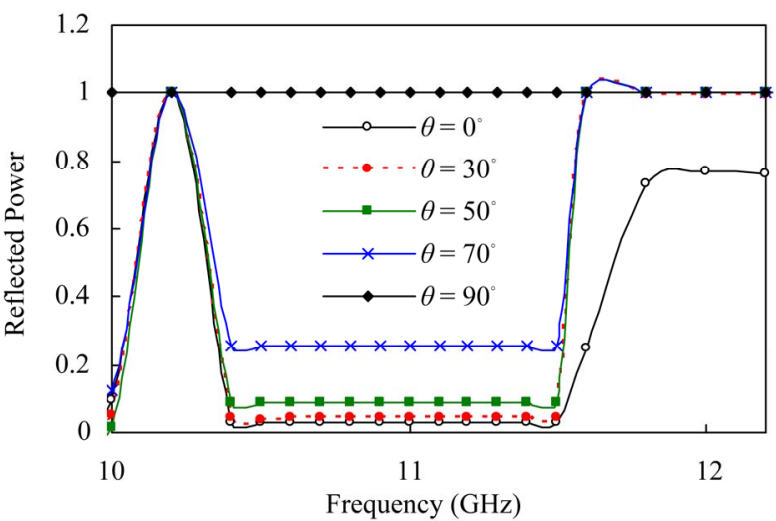

(a)

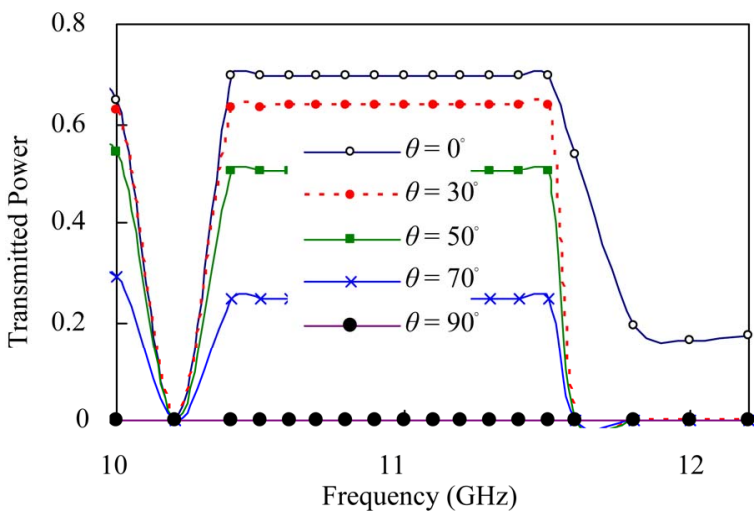

(b)

Figure 5. The reflected and transmitted powers against frequency when $n_{1} \neq n_{4}, n_{2}=\left|n_{3}\right|$ and $a=d$ for various angle of incidences: $\theta$ $=0^{\circ}, \theta=30^{\circ}, \theta=50^{\circ}, \theta=70^{\circ}, \theta=90^{\circ}$.

has been satisfied by the obtained results. The dependence of propagation on the given parameters gives rise to possibilities of tuning the transmitted power for applications in microwave, antenna radome, millimeter wave, 


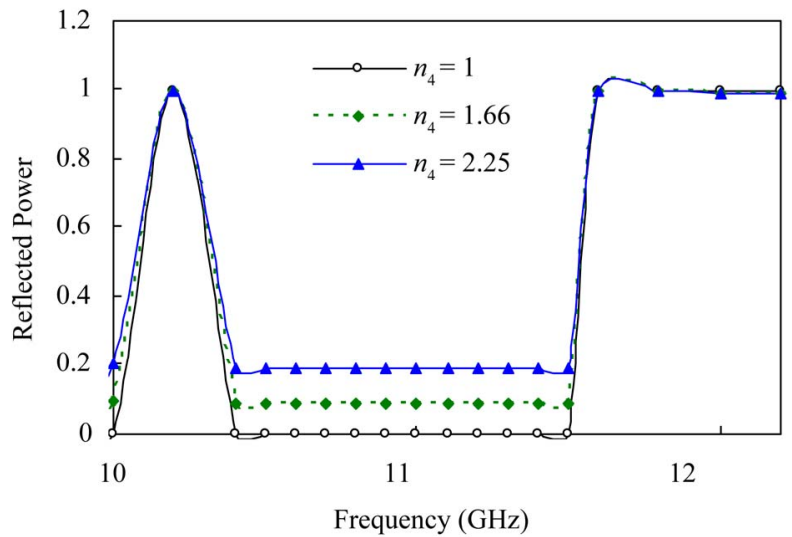

(a)

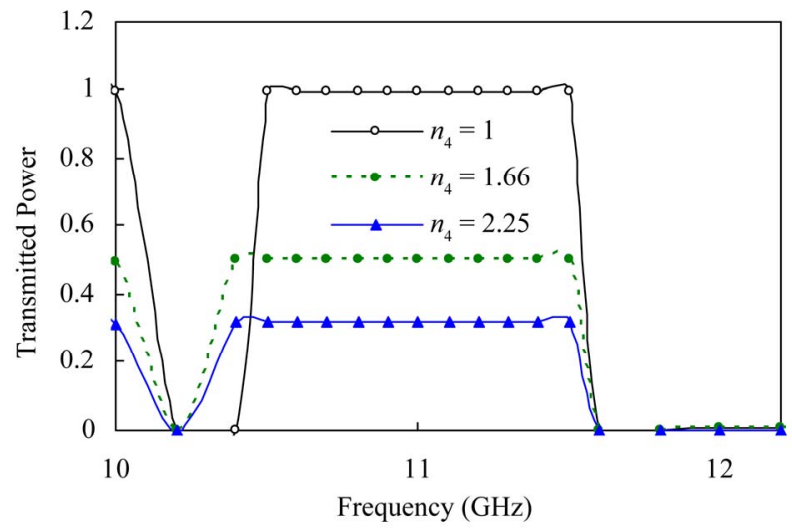

(b)

Figure 6. The reflected and transmitted powers versus frequency when $n_{1} \neq n_{4}, n_{2}=\left|n_{3}\right|$ and $a=d$, and the angle of incidence $\theta=30^{\circ}$ (kept constant) for different value of $n_{4}: n_{4}=1, n_{4}=1.66, n_{4}=2.25$.

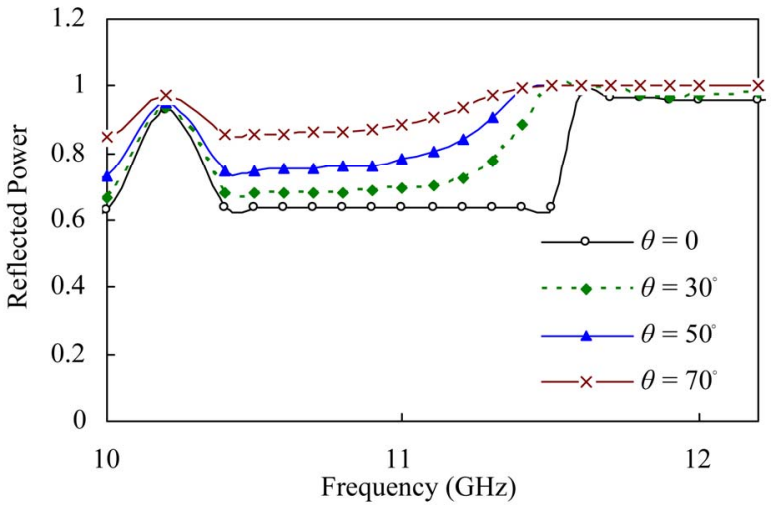

(a)

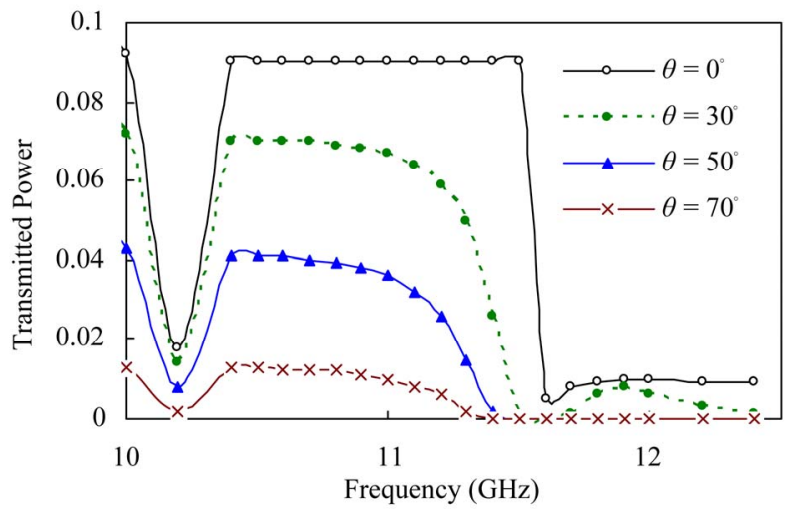

(b)

Figure 7. The reflected and transmitted powers as a function of frequency where the condition $\left(n_{1}<n_{2}, n_{2}>n_{3}, n_{3}<n_{4}\right)$ is satisfied for various angle of incidences: $\theta=0^{\circ}, \theta=30^{\circ}, \theta=50^{\circ}, \theta=70^{\circ}$.

and optical devices.

\section{REFERENCES}

[1] Veselago, V.G. (1986) The electrodynamics of substances with simultaneously negative values of $\varepsilon$ and $\mu$. Soviet Physics Uspekhi, 10, 509-514.

doi:10.1070/PU1968v010n04ABEH003699

[2] Pendry, J.B., Holden, A.J., Sewart, W.J. and Youngs, I. (1996) Extremely low frequency plasmons in metallic meso- structure. Physics Review Letters, 76, 4773-4776. doi:10.1103/PhysRevLett.76.4773

[3] Pendry, J.B., Holden, A.J., Robbins, D.J. and Stewart, W.J. (1999) Magnetic from conductors and enhanced nonlinear phenomena. IEEE Transaction on Microwave Theory and Techniques, 47, 2075-2084. doi:10.1109/22.798002

[4] Shelby, R.A., Smith, D.R. and Schultz, S. (2001) Experimental verification of a negative index of refraction. Science, 292, 77-79. doi:10.1126/science.1058847

[5] Shadrivov, I.V. (2004) Nonlinear guided waves and symmetry breaking in left-handed waveguides. Photonic Nanostruct: Fundamentals and Application, 2, 175-180.

[6] Garcia, N. and Nieto-Vesperinoas, M. (2002) Left-handed materials do not make a perfect lens. Physical review letters, 88, 7403.

doi:10.1103/PhysRevLett.88.207403

[7] Kong, J.A. (2002) Electromagnetic wave interaction with stratified negative isotropic media. Progress in Electromagnetic Research, 35, 1-52. doi:10.2528/PIER01082101

[8] Mousa, H.M. and Shabat, M.M. (2007) Nonlinear TE surface waves in a left-handed material and magnetic super lattice waveguide structure. Modern Physics B, 21, 895-906. doi:10.1142/S0217979207036746

[9] Kourakis, I. and Shukla, P.K. (2005) Nonlinear propagation of electromagnetic waves in negative-refraction-index composite materials. Physical Review E, 72, 016626. doi:10.1103/PhysRevE.72.016626

[10] Cory, H. and Zach, C. (2004) Wave propagation in metamaterial multi-layered structures. Microwave and $\mathrm{Op}$ tical Technology Letters, 40, 460-465. doi:10.1002/mop.20005 
[11] Sabah, C. and Uckun, S. (2009) High reflection coating with negative and positive refractive index. Piers Online, 5, 601-604. doi:10.2529/PIERS090220061404

[12] Oraizi, H. and Abdolali, A. (2009) Mathematical formulation for zero reflection from multilayered metamaterial structures. IET Microwaves Antennas and Propagation, 3, 987-996. doi:10.1049/iet-map.2008.0281

[13] Stancil, D.D. and Prabhakar, A. (2009) Spin waves. Springer Science, New York.

[14] Sabah, C. and Uckun, S. (2007) Electromagnetic wave propagation through frequency-dispersive and lossy double-negative slab. Opto-Electronics Review, 15, 133-134. doi:10.2478/s11772-007-0011-yv
[15] Shelby, R.A. (2001) Microwave experiments with left-handed materials. PhD. Dissertation, University of California, San Diego.

[16] Pendry, J.B., Holden, A.J., Robbins, D.J. and Stewart, W.J. (1998) Low frequency plasmons in thin wire structures. Condensed Matter Physics, 10, 4785-4809. doi:10.1088/0953-8984/10/22/007

[17] Sievenpiper, D.F., Yablonovitch, E., Fan, J.N., Villeneuve, P.R. and Joannopoulos, J.D. (1998) 3D metallo-dielectric photonic crystals with strong capacitance coupling between metallic islands. Physical Review Letters, 80, 2829-2832.doi:10.1103/PhysRevLett.80.2829 